\title{
Dolor en personas adultas con un trastorno del espectro del autismo (TEA) y comorbilidad: un análisis mediacional
}

\author{
Pain in adults with autism spectrum disorders and comorbidity: \\ a mediational analysis
}

\section{Resumen}

La literatura incluyó las anomalías del procesamiento sensorial (dolor asociado) en los criterios de diagnóstico para las personas con autismo (TEA). Sin embargo no disponemos de ningún método estándar para la evaluación de las anomalías sensoriales y la conducta de dolor en personas con TEA. Cuarenta y seis adultos con TEA fueron incluidos en este estudio. Se evaluó la sensibilidad al dolor procedimental y agudo de forma objetiva en diversos contextos. Luego analizamos la matriz de correlación y ejecutamos un análisis dimensional. La correlación entre la sensibilidad al dolor y las variables clínicas fue significativa. La comorbilidad fue el principal factor de mediación entre la severidad del autismo y la intensidad del dolor. Nuestros resultados sugieren que nuestra metodología puede ser utilizada para evaluar la sensibilidad al dolor en adultos con TEA y nosotros confirmamos la hipótesis de que la comorbilidad es el principal factor de mediación entre la severidad del autismo y la intensidad del dolor.

\section{Palabras clave}

Adultos con TEA, anormalidad sensorial, dolor, comorbilidad del autismo.

\begin{abstract}
The literature recently included sensory processing abnormalities (pain associated) in the diagnostic criteria for individuals with autism spectrum disorder (ASD). However, there is no standard method for evaluating sensory and pain abnormalities in individuals with ASD. Forty-six adults with ASD were enrolled in this study. We evaluated objective pain sensitivity by measuring the procedural and acute pain in several contexts. Then, we analyzed the correlation matrix and next we executed mediational analyzes. The correlation between perception of pain and clinical variables was significant. The comorbidity was the main factor in the mediation between the severity of autism and the intensity of pain. Our results suggest that our methodology can be used to assess pain sensitivity in individuals with ASD, and we confirmed the hypothesis that comorbidity is the main mediating factor between the severity of autism and the intensity of pain.
\end{abstract}

\section{Keywords}

Adults with autism spectrum disorder (ASD), sensory abnormality, pain, comorbidity.

\section{Domingo García-Villamisar \\ <villamis@edu.ucm.es>}

Universidad Complutense de Madrid
Para citar:
García-Villamisar, D. (20I7):
"Dolor en personas adultas con un
trastorno del espectro del autismo
(TEA) y comorbilidad: un análisis
mediacional". Revista Española de
Discapacidad, 5 (2): 73-86.

Doi: <https://doi.org/IO. 5569/23405 I04.05.02.04>

Fecha de recepción: 20-02-2017 Fecha de aceptación: I7-IO-20I7 


\section{Introducción 1}

Los trastornos del espectro del autismo (TEA) en personas adultas están alcanzando una mayor relevancia en el ámbito de los cuidados de la salud, tal como demuestran los datos epidemiológicos de reciente aparición que nos informan de un incremento espectacular de la prevalencia de este trastorno, en parte debido a la naturaleza espectral que adquirió el trastorno de acuerdo a la nueva clasificación de la DSM-5 (APA, 20I3). Conforme a este sistema de clasificación el espectro del autismo se caracteriza por la presencia de unos déficits crónicos para la comunicación interpersonal y un comportamiento repetitivo y estereotipado. Además el autismo es un trastorno crónico del que se han hecho pocos estudios de seguimiento en la vida adulta (para una revisión, Howlin y Magiati, 20I7).

En todo caso, se puede afirmar que, aunque las habilidades adaptativas permanecen estables en la vida adulta (Fusar-Poli et al., 20I7), sólo una minoría logra una vida más o menos independiente (Grinker, 2015; Howlin y Magiati, 20I7). La prevalencia del trastorno es mucho más elevada que antaño, con una afectación en torno al i \% de la población, aunque se cree que la mayoría están sin diagnosticar, pues las personas mayores fueron evaluadas con criterios mucho más restrictivos para el diagnóstico del autismo (Brugha, 20I6), con una prevalencia de 3:I en varones frente a las mujeres (Loomes et al., 20I7). Esto supone que, extrapolando los datos, unos 52 millones de personas en el mundo sufren este trastorno.

Por ello se observa un renovado interés por la vida adulta, tal como ponen de relieve algunos trabajos recientes (Bishop-Fitzpatrick et al., 201 5; Happé y Charlton, 20I 2; Howlin y Moss, 20I 2; Howlin y Magiati, 20I7; Mazefsky y

I. Financiación: este artículo proviene de un proyecto de investigación financiado por el Real Patronato sobre Discapacidad en 2015 .

Agradecimientos: el autor agradece a Carmen Muela y al equipo técnico de la Asociación Nuevo Horizonte la coordinación práctica de este proyecto y la colaboración que en todo momento fue necesaria para el desarrollo del mismo.
White, 20I4; Shattuck et al., 20I2; Tantam, 20I4). Esta tendencia se refleja en los cuidados de la salud (Croen et al., 20I 5; Warfield et al., 20I 5), la psicopatología (García-Villamisar y Rojahn, 201 5), el derecho a la educación continua y al empleo (Della Fina y Cera, 20 I 5 ) y una investigación cada vez más intensa y fructífera (Piven y Rabins, 20II).

Uno de los componentes esenciales de la calidad de vida en los adultos con autismo y, en general en las personas mayores, es una salud óptima. Disponer de una buena salud mejora sustancialmente la calidad de vida y disminuye las tasas de mortalidad (Brown et al., 20I 5 ). Por otra parte se ha demostrado que los adultos con autismo presentan una mayor incidencia de problemas psiquiátricos comórbidos, tales como ansiedad, depresión, trastorno bipolar, trastorno obsesivo-compulsivo e intentos de suicidio que la población general. Igualmente, la mayor parte de las enfermedades afectan más frecuentemente a los adultos con autismo, tales como enfermedades inmunes, trastornos gastrointestinales y del sueño, epilepsia, obesidad, dislipemia, hipertensión, diabetes, etc., a parte de las enfermedades comunes, tales como gripe, traumatismos, etc., tal como puso de manifiesto la reciente revisión de Croen et al. (2OI 5 ) realizada con una amplia muestra de I 500 personas con autismo. Cuando se sufre alguna estas enfermedades el denominador común es el dolor y el consiguiente malestar. La identificación y abordaje del dolor en la práctica clínica debería ser uno de los elementos de apoyo nucleares para las personas con TEA.

El dolor fue definido por la Asociación Internacional para el Estudio del Dolor (International Association for the Study of Pain, IASP) como "an unpleasant sensory or emotional experience associated with actual or potential tissue damage or described in terms of such damage” (Latham y Davis, I994). El dolor puede tener un efecto muy negativo en la habilidad funcional, la movilidad, el status

2. Traducción del editor: una experiencia sensorial o emocional desagradable asociada a un daño real o potencial en un tejido, o descrita en términos de ese daño. 
emocional o en las actividades sociales de las personas con autismo, lo cual determinará un uso progresivo de los servicios de salud y un incremento del coste asistencial de estas personas. Además, dado que la mayoría de las personas con autismo carecen de lenguaje, la identificación del dolor por parte de los profesionales de la salud constituye un auténtico reto.

La identificación y evaluación del dolor en estos pacientes puede realizarse a diferentes niveles: fisiológico (a través de la tasa cardíaca o de la respiración), conductual (manifestaciones no verbales y verbales del dolor, tales como gestos, inquietud, llanto, autoagresiones, etc., y que pueden ser valoradas por observadores (Van der Putten y Vlaskamp, 20I I).

La evaluación del dolor en personas con TEA es compleja no sólo por las dificultades de comunicación que presentan, sino también por su discapacidad intelectual asociada y la peculiaridad con la que estas personas perciben la experiencia dolorosa. Aunque las personas con autismo presentan una percepción sensorial alterada (Baum et al. 20 I 5; Moore, 201 5), esta anomalía sensorial ha permanecido largo tiempo sin ser reconocida (Duerden et al., 20I 5 ) y sólo recientemente el DSM-5 (APA, 20I3) la incorporó como un criterio diagnóstico. La percepción sensorial anómala del dolor puede ser consecuencia tanto de la hipersensibiliad como de la hiposensibiliad ante la estimulación dolorosa. La hipersensibiidad se manifiesta porque las personas más sensibles al dolor muestran unas conductas muy exageradas ante una estimulación dolorosa que en general se juzga como benigna. Estas manifestaciones suelen acompañarse de evidentes signos de ansiedad y agresividad (Roth, 2010). Algunas de las manifestaciones conductuales asociadas a esta hipersensibildad son, por ejemplo, taparse oídos ante una señal ruidosa poco intensa, evitar la mirada, etc. Por el contrario, las personas con TEA que son hiposensibles al dolor, muestran una notable indiferencia a la estimulación nociva, por lo que se ven frecuentemente avocados a situaciones de peligro (Kern et al., 2006). La hiposensibilidad al dolor se manifiesta por una respuesta débil ante situaciones aversivas tales como el frío o el calor (APA, 2013) o las autolesiones (Oliver et al., 2017).

De la literatura publicada se desprende que el dolor es un ejemplo típico de la hiposensibilidad de las personas con TEA a las señales nocivas, pues tanto las historias de caso como los informes médicos y de las propias familias refieren la extraña hiposensiblidad que muestran estas personas (Moore, 20I5). Un porcentaje superior al $70 \%$ de las personas con autismo presentan con mucha frecuencia estereotipias y autolesiones, lo que ha llevado a sospechar de una baja sensibilidad al dolor por parte de estas personas.

Entre los estudios que han corroborado la hiposensibilidad al dolor de las personas con TEA merecen citarse el realizado por Minshew y Hobson (2008), quienes a través de medidas neurofisiológicas y cuestionarios demostraron que dos tercios de las personas con autismo investigadas fueron más sensibles al dolor que el grupo normotípico. Militerni et al. (2010) pusieron de relieve la reactividad anormal al dolor de las personas con TEA. En su estudio encontraron que un $22 \%$ de los participantes en su muestra de estudio mostraba una baja sensibilidad al dolor y un 2 I \% una sensibilidad muy baja. Klintwall et al. (2OI I) demostraron que el $40 \%$ de los sujetos estudiados presentaba una baja reactividad al dolor, aunque la respuesta al dolor difería entre los diversos subgrupos de personas con autismo. Gillberg et al. (I985) observaron en su estudio que más de la mitad de la muestra estudiada presentaba hiposensiblidad al dolor. Fan et al. (2013) y Cascio et al. (2008) pusieron de relieve en sus respectivos estudios una menor sensibilidad al dolor en el grupo de las personas con autismo que los controles.

Por el contrario, otros estudios no encontraron diferencias entre las personas con autismo y los controles, tales como Bandstra et al. (2OI2), Bird et al. (2010) o Tordjman et al. (2009). En el polo opuesto encontramos el trabajo de Nader et al. (2004) donde probaron que el grupo de autismo fue más sensible al dolor 
que el grupo de control y Hechler et al. (2016) demostraron el por qué ciertas sensaciones inocuas son percibidas como aversivas debido al condicionamiento interoceptivo.

En síntesis, estos estudios refieren una tendencia más o menos universal a la hiposensiblidad al dolor por parte de las personas con autismo, aunque cabrían otras explicaciones alternativas, tales como la dificultad para expresar o comunicar las sensaciones dolorosas, su intensidad o su calidad (Moore, 201 5; Rattaz et al., 2016). En esta línea argumental, Yasuda et al. (20I6) postulan que las personas con TEA sufren un deterioro cognitivo que les impide procesar correctamente la señal dolorosa.

Por ello, uno de los problemas más urgentes a resolver en este contexto es la identificación del dolor de la forma más acertada y precisa posible a través de nuevos procedimientos, a falta de instrumentos adecuados al respecto, pues la mayor parte de las personas con autismo carecen de lenguaje. Este problema es más acuciante, si cabe, en el grupo de personas adultas con autismo, pues no se han desarrollado instrumentos de diagnóstico del dolor específicos, por lo que la mayor parte de las situaciones de dolor están escasamente identificadas, al menos en los comienzos del problema, ya que las personas con autismo no aciertan a concretar el foco de su dolor.

A la vista de la respuesta tan desigual que ofrecen las personas con autismo ante la misma señal dolorosa, cabe preguntarse qué papel juegan ciertas variables transdiagnósiticas como potenciales mediadores de la respuesta a la intensidad del dolor en las personas con TEA. En el siguiente apartado realizamos una breve revisión de esta potencial relación y planteamos las hipótesis a desarrollar.

\section{Mediadores del dolor en el autismo: comorbilidad, estrés y disfunciones ejecutivas}

La comorbilidad es muy frecuente entre las personas adultas con autismo. Por lo que se refiere a la depresión, los estudios epidemiológicos informan de una elevada comorbilidad entre la depresión y el autismo abarcando desde el I, $4 \%$ al $70 \%$ (Postorino et al., 2016), la cual es muy difícil de establecer con precisión, pues son muy diferentes los criterios de diagnóstico utilizados y los motivos de referencia.

En general, la literatura establece una relación recíproca entre depresión y dolor (Thompson et al., 2016). La depresión dificulta el abordaje terapéutico del dolor y los pacientes con dolor presentan más quejas de dolor, mayor intensidad, más duración de la señal dolorosa y un peor pronóstico en cuando a la recuperación. La depresión también predice un mayor uso de los cuidados de la salud y una peor adherencia a los tratamientos.

La ansiedad y el autismo están interrelacionados conceptualmente desde la acuñación del término autismo en tiempos de Kanner. La ansiedad interfiere en la mayor parte de los ámbitos de las personas con autismo, tales como las relaciones sociales, el ocio, la calidad de vida o los problemas médicos, incluido el dolor (Uljarevic et al., 2016). La presencia de la ansiedad en las personas con autismo afecta a una amplia proporción de casos que algunos autores estiman en sus revisiones entre el I3,6 y el 84, I \% (Uljarevic et al., 2016). Hay una evidencia clínica cada vez más creciente de que el dolor, especialmente el dolor crónico, ocurre conjuntamente con los trastornos de la ansiedad. Esta concurrencia es desatendida en la práctica clínica porque no hay protocolos estándar en el contexto del autismo que identifiquen esta relación. Además, cuando se presentan de forma conjunta ambos trastornos, provocan un gran malestar en estas personas. Por ello, la evaluación y el tratamiento de ambos trastornos es complicada. 
Por lo que se refiere al estrés, hay una significativa relación entre estrés y dolor (Hufnagel et al., 20I7). Se puede observar que en muchos casos el estrés obedece a la dificultad que presentan las personas con TEA para comunicarse e interaccionar. Aunque no hay una medida precisa del estrés en adultos con autismo, ciertos autores (Bishop-Fitzpatrick et al., 2015) utilizan de forma sistemática los cuestionarios y entrevistas, aunque no se apoyan en medias fisiológicas, por lo que estos resultados hay que aceptarlos con cierta precaución. En todo caso, se observa la necesidad de medias específicas para evaluar el estrés en personas adultas con autismo (Hufnagel et al., 2017).

Las disfunciones ejecutivas afectan al trastorno autista a lo largo de todo el ciclo life-span (Demetriou et al., 20I7). En relación con el dolor, hay un acuerdo general con respecto al papel que juega el control de la atención -una dimensión de las funciones ejecutivas- en la experiencia dolorosa (Eccleston, I995). Sin embargo, algunos estudios más recientes proponen que otras funciones ejecutivas pueden jugar un rol importante en la respuesta al dolor (Abeare et al., 2010), aunque hay aspectos conceptuales que arrojan cierta oscuridad en esta relación (Bento, et al., 2017; Nes et al., 2009). Algunos estudios probaron que una dimensión de las funciones ejecutivas, la inhibición, estaba asociada a una menor sensibilidad al dolor (Oosterman et al., 2010) y a una mayor tolerancia al dolor (Karsdorp et al., 2013), aunque no se sabe si esta asociación está vinculada sólo a la inhibición, o participan también otras funciones ejecutivas, si bien Oosterman et al. (20I0) incluyeron otras funciones ejecutivas y no encontraron relación con medidas experimentales del dolor. Sin embargo, este planteamiento plantea algunos interrogantes. En primer término, las funciones ejecutivas abarcan un amplio espectro y no hay un acuerdo para categorizar empíricamente esta diversidad de funciones (Friedman y Miyake, 20I7). En segundo lugar, la diversidad de funciones ejecutivas dificulta establecer una relación unidimensional con el dolor, razón por la que parece oportuno optar por medidas ecológicas de las funciones ejecutivas que consideren a estas desde un punto de vista holístico. En tercer lugar, aunque no lo abordaremos aquí por exceder el propósito de este artículo, habría que tener en cuenta otras variables mediadoras, tales como la medicación o el sueño, a la hora de analizar los factores mediadores entre la severidad del autismo y la percepción del dolor (Masiliūnas et al., 20I7).

Por ello, el objetivo de este artículo está orientado a identificar el dolor en personas adultas con autismo y a valorar el impacto mediacional que pudiera tener la psicopatología comórbida, el estrés y las disfunciones ejecutivas. De acuerdo a la literatura publicada, se pretende, por lo tanto, demostrar las siguientes hipótesis:

I. En el contexto clínico y durante el período de observación establecido, la respuesta al dolor correlacionará de forma positiva y significativa con la psicopatología comórbida (sintomatología ansiosodepresiva), las disfunciones ejecutivas y el nivel de estrés.

2. La relación entre las conductas de dolor y la severidad del trastorno del autismo estará mediada por la psicopatología comórbida (sintomatología ansioso-depresiva), el estrés y las disfunciones ejecutivas en la vida diaria.

\section{Metodología}

\subsection{Participantes}

La muestra de participantes es de conveniencia por razones clínicas y de proximidad con el investigador, y está constituida por 46 personas adultas con autismo de ambos sexos (30 varones y I 6 mujeres) pertenecientes a la Asociación Nuevo Horizonte de las Rozas (Madrid), un centro especializado en la rehabilitación de personas adultas con autismo. La edad media fue de 37.2 años (DT =7.84; rango 20-48). Todos los participantes tienen una discapacidad intelectual de tipo moderado y la mayoría 
carecen de lenguaje. Los participantes fueron observados durante determinadas situaciones cotidianas de dolor: vacunas y analíticas, tratamiento e higiene dental y diversas situaciones dolorosas, tales como dolor de cabeza, de garganta, traumatismos, esguinces, etc.

\subsection{Materiales e instrumentos}

\subsubsection{Instrumentos}

Para la evaluación objetiva del dolor, se utilizó el siguiente instrumento:

- Non-Communicating Adult Pain Checklist (NCAPC; Lotan et al., 2010).

NCAPC es una escala de 18 ítems que se puntúa en una escala de o a 3 , referido a la duración de la conducta observada. Evalúa 6 subdimensiones del dolor: reacción vocal, reacción emocional, expresión facial, lenguaje corporal, reacción de autoprotección y reacción fisiológica. La puntuación se basa en las conductas del dolor observadas durante un periodo de tiempo definido. La puntuación se realizó con arreglo a los datos videograbados y/o observados de cada situación de dolor sufrida por cada participante. La consistencia interna de esta escala fue aceptable (Cronbach's $\alpha$ de Cronbach $=$ 0.72) (Lotan et al., 20IO). El doctor Lotan autorizó al director de este proyecto la adaptación de este cuestionario al ámbito del autismo (García-Villamisar et al., 2017).

- Autism Spectrum Disorders Diagnostics for Adults (ASD-DA; Matson et al., 2008).

La escala ASD-DA fue diseñada por el grupo de Matson para el diagnóstico del trastorno del autismo en la vida adulta. Los ítems del cuestionario recogen la sintomatología nuclear de los TEA de acuerdo a los criterios de los actuales sistemas de Clasificación DSM-IV (American Psychiatric Association, 2000) y CIE-Io (World Health Organization, I992) y de otras escalas frecuentemente utilizadas en el ámbito del autismo, tales como la Childhood Autism Rating Scale (CARS) y la subescala de autismo del Diagnostic Assessment for the Severely HandicappedII (DASH-II) (Matson et al., 2008). Este cuestionario tiene 3 I ítems que evalúan los síntomas principales del autismo en adultos. Cada ítem se puntúa con arreglo a dos posibles valores, 'No es diferente, no hay discapacidad' (o) o 'Diferente, con discapacidad' (I). Algunos ítems incluidos son, 'Habilidades de autonomía y adaptación propias de su edad (o sea, si es capaz de cuidar de sí mismo)', 'Capacidad de reconocer las emociones de los demás', 'uso del lenguaje para comunicarse', 'Comparte diversión, interés o éxito con los demás (padres, amigos, cuidadores)', 'Le interesan pocas cosas', 'Participación en juegos u otras actividades sociales'. Este cuestionario goza de buenas propiedades psicométricas ( $\alpha$ de Cronbach $=0.94$; Matson et al. 2008).

- $\quad$ Autism Spectrum Disorder Comorbility for Adults (ASD-CA; Matson et al., 2008).

La escala ASD-CA (Matson et al., 2007), es una escala de 37 ítems para la valoración de la comorbilidad. Los ítems están valorados con tres criterios, 'No es en absoluto un problema ni una discapacidad' (o), 'Problema o discapacidad leve' (I) y 'No se aplica o no lo sabe' (X). Algunos de estos ítems son 'Se enfada con facilidad', 'Come demasiado rápido’, etc. El cuestionario ASD-CA tiene una aceptables propiedades psicométricas con una moderada fiabilidad test-retest $(\mathrm{k}=0.59)$ y una muy buena consistencia interna ( $\alpha=0.9 \mathrm{I}$ ) (Matson et al., 2008). Este cuestionario evalúa la sintomatología propia de los siguientes factores: ansiedad/conductas repetitivas, problemas de conducta, irritabilidad, déficit de atención/impulsividad y humor depresivo (Matson et al., 2008; LoVullo y Matson, 2009).

- The Stress Survey Schedule for Persons with Autism and Other Pervasive Developmental 


\section{Disabilities (Stress SS; Groden et al., 2006).}

La Stress-SS fue desarrollada para evaluar el estrés en la vida de las personas con autismo y ayudar a identificar qué situaciones causan respuestas de estrés en estas personas (Groden et al., 2006). Groden et al. (200I) aplicaron un análisis factorial confirmatorio y demostraron que la Stress-SS tiene una estructura interna configurada por ocho factores que son muy relevantes en ámbito del autismo. En esta investigación se utilizó la puntuación total de la escala como un índice global del estrés. En consonancia con el procedimiento de administración descrito por Groden et al. (200I), si los participantes poseen un adecuado nivel de lenguaje receptivo y expresivo, cumplimentarán directamente la escala. En caso contrario, serán los terapeutas muy próximos al sujeto quienes proporcionen esta información. Las cualidades psicométricas son muy aceptables (Groden et al., 200I).

- $\quad$ Dysexecutive Questionnaire (DEX; Burguess et al., 1998).

El Dysexecutive Questionnaire (DEX) es un autoinforme de 20 ítems destinado a evaluar los síntomas de la disfunción ejecutiva en la vida diaria (Burguess et al., I998). El DEX (versión informe del observador) requiere a los evaluadores puntuar en una escala tipo Likert entre 0 (nunca) y 4 (muy a menudo) cuán a menudo se observan cada uno de los 20 problemas ejecutivos recogidos en la escala. Cuanto más elevada sea la puntuación en la escala, más disfuncional es la conducta ejecutiva de los pacientes. La fiabilidad es adecuada ( $\alpha=$.90; Burguess et al., I 998). En esta investigación se utilizará la puntuación total de la escala. Este cuestionario fue incluido en esta investigación tanto por su naturaleza ecológica como porque ofrece una valoración holística de las funciones ejecutivas en la vida diaria.

\subsection{Procedimiento}

Se seleccionó a un grupo de terapeutas de la institución con gran conocimiento de los participantes y se les formó en la identificación del dolor a través del instrumento NonCommunicating Adult Pain Checklist (NCAPC; Lotan et al., 20IO). Tal como se describió en el apartado anterior, el NCAPC es uno de los instrumentos más adecuados para identificar el dolor en personas adultas con discapacidad, aunque no es específico del autismo. El autor principal obtuvo la autorización del doctor Lotan para la adaptación de este instrumento al español y para su aplicación a personas con autismo. Se impartieron una serie de sesiones formativas a los terapeutas participantes en el estudio para explicarles la metodología de observación del dolor, aunque manteniéndolos en todo caso ciegos a los objetivos finales del estudio. La fiabilidad interjueces evaluada a través del coeficiente Kapa de Kohen fue de .82. Los observadores estaban constituidos por los tutores de los participantes, por su conocimiento próximo de los destinatarios.

\subsection{Análisis de datos}

Se realizaron análisis de correlación entre las diversas variables para estudiar la interrelación entre las mismas. Igualmente se realizaron análisis de regresión y mediación para estudiar el efecto modulador de la psicopatología comórbida, el estrés y las disfunciones ejecutivas en la percepción de la conducta del dolor. Para ello se utilizó el programa SPSS y el programa Process de Hayes (2013).

\section{Resultados}

En este apartado se describen los resultados alcanzados en esta investigación. 


\subsection{Análisis correlacional entre la percepción del dolor y la psicopatología (variables clínicas)}

En la Tabla I se exponen los resultados correspondientes al análisis de correlación entre las diversas variables incluidas en el estudio.

Tal como se aprecia en la Tabla I, la puntuación total de la respuesta al dolor correlaciona de forma positiva y significativa con la sintomatología del autismo (ASD-D) ( $r=.365$; $\mathrm{p}<$.OI), la comorbilidad asociada al trastorno autista (ASD-CA) $(r=.433$; $\mathrm{p}<$.oI) y con la disfunción ejecutiva en la vida diaria (DEX) (r $=.3$ I $4 ; \mathrm{p}<.05)$. Se confirma así parcialmente la primera hipótesis, ya que predecía que la respuesta al dolor correlacionaría de forma positiva y significativa con la sintomatología del autismo, la comorbilidad y la disfunción ejecutiva. No obstante, la respuesta al dolor y el estrés no correlacionaron de forma significativa.

\subsection{Estudio mediacional}

El modelo 4 del macro Process fue utilizado para determinar si los efectos indirectos (mediación múltiple) relativos a las 3 variables comorbilidad, estrés y disfunción ejecutiva en la vida diaria son significativos en la mediación

Tabla 1. Correlación $\mathrm{r}$ de Pearson entre el dolor y las variables psicopatológicas analizadas

\begin{tabular}{|l|c|c|c|c|c|c|}
\hline & $\mathbf{2}$ & $\mathbf{3}$ & $\mathbf{4}$ & $\mathbf{5}$ & $\overline{\mathbf{x}}$ & $\mathbf{D T}$ \\
\hline 1. NCAPC & $.365^{*}$ & $.433^{* *}$ & .237 & $.314^{\star}$ & 18.190 & 6.701 \\
\hline 2. ASD-D & & $.703^{* *}$ & $.606^{* *}$ & $.736^{\star *}$ & 14.278 & 3.339 \\
\hline 3. ASD-CA & & & $.580^{* *}$ & $.675^{\star \star}$ & 22.200 & 4.225 \\
\hline 4. Stress & & & & $.577^{\star *}$ & 110.700 & 9.732 \\
\hline 5. DEX & & & & & 40.000 & 7.592 \\
\hline
\end{tabular}

$* \mathrm{p}<.05 ; *$ p $<.0 \mathrm{I}$

I. Non-Communicating Adult Pain Checklist (NCAPC; Lotan et al., 20I0); 2. Autism Spectrum Disorders Diagnostics for Adults (ASD-D; Matson et al., 2008); 3. Autism Spectrum Disorder Comorbility for Adults (ASD-CA; Matson et al., 2008); 4. The Stress Survey Schedule for Persons with Autism and Other Pervasive Developmental Disabilities (Stress SS; Groden et al., 2006); 5. Dysexecutive Questionnaire (DEX; Burguess et al., 1998).

Nota: se incluye la media $(\bar{x})$ y la desviación típica $(D T)$ de cada una de las variables incluidas en el análisis.

Fuente: elaboración propia.

Tabla 2. Coeficientes del modelo

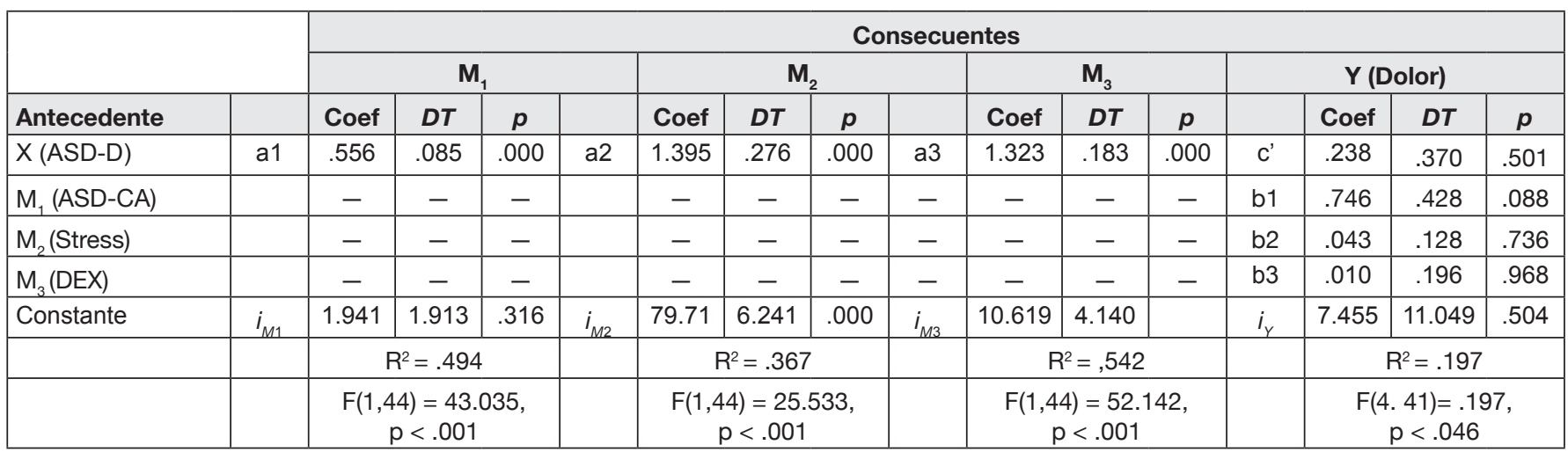

Fuente: elaboración propia. 
entre la severidad de la sintomatología del autismo y la puntuación total de dolor. El modelo de mediación múltiple en el cual todas las variables fueron introducidas simultáneamente permitió la investigación de los efectos indirectos de las diferentes escalas. Las Tablas 2 y 3 muestran los resultados relativos al análisis mediacional entre la sintomatología del autismo y la respuesta al dolor. De acuerdo a las datos de la Tabla 2, la sintomatología del autismo no presentó un efecto directo en la percepción del dolor $\left(\mathrm{c}^{\prime}=.238, \mathrm{p}<.5 \mathrm{OI}\right)$, aunque sí se observó el efecto indirecto relativo a la comorbilidad (ASD-CA), pues no aparece una puntuación cero entre Lower limit (LLCI) del intervalo de confianza y el upper limit (UUCI) o límite superior del intervalo de confianza (95\% UUCI-ULCI) (.02-I,02) (Tabla 3). Los demás efectos indirectos no son significativos.

\begin{tabular}{|c|c|c|c|}
\hline & $\begin{array}{c}\text { Efecto } \\
\text { indirecto }\end{array}$ & LLCI & ULCI \\
\hline Comorbilidad & .4292 & .0250 & 1.0182 \\
\hline Estrés & .0402 & -.4085 & .7532 \\
\hline $\begin{array}{l}\text { Disfunción } \\
\text { ejecutiva }\end{array}$ & .1064 & -.0271 & .4390 \\
\hline
\end{tabular}

Fuente: elaboración propia.

\section{Discusión y conclusiones}

El presente estudio investigó en qué medida la percepción del dolor por parte de las personas adultas con autismo con problemas médicos ordinarios está mediado por la psicopatología comórbida, el estrés y las disfunciones ejecutivas en la vida diaria. La evaluación del dolor en el ámbito del autismo, en particular con las personas con autismo no verbales, es uno de los retos más importantes en los centros del autismo. Las personas con autismo sufren algunas alteraciones médicas que requieren unos procedimientos exploratorios y terapéuticos dolorosos. Además, la incapacidad de estas personas para verbalizar la experiencia dolorosa supone a veces una terapéutica inapropiada.

Hay diferentes tipos de dolor, por ejemplo dolor agudo, dolor crónico, dolor recurrente, dolor asociado a enfermedad terminal y dolor procedimental relacionado con el abordaje terapéutico de los problemas de salud cotidianos, tales como vacunas y analíticas, tratamientos e higiene dental y situaciones dolorosas habituales como dolor de garganta, dolor de cabeza, pequeños traumatismos, esguinces, etc. El dolor procedimental es específico en la medida en que los pacientes pueden anticipar esa situación dolorosa por malas experiencias previas, por lo que a su vez la activación emocional por vía de la anticipación puede jugar un papel importante en la modulación de la experiencia dolorosa y en el incremento del malestar. Por otra parte, debido a que las personas adultas con autismo suelen tener una historia médica con diversos problemas, se incrementa la probabilidad de que previamente haya sufrido situaciones adversas en el ámbito del tratamiento.

Esta investigación puso de manifiesto una elevada correlación entre la intensidad del dolor y la severidad del autismo. Al mismo tiempo se puso de relieve una relación estadísticamente significativa entre la intensidad del dolor percibido y la psicopatología comórbida y la disfunción ejecutiva en la vida diaria, pero esta relación no se mantuvo en relación con el estrés.

La relación entre el dolor y la función ejecutiva en general (Moriarty et al., 20I I) y en la vida diaria (Baker et al., 2016) está ampliamente demostrada, en coincidencia con lo demostrado en esta investigación. Al mismo tiempo, la relación entre el dolor y la psicopatología comórbida fue probada en esta investigación en consonancia con otras publicaciones recientes (Boucher, 20I7; Mannix, 20I6).

Pero el hallazgo más relevante de esta investigación es la mediación de la 
sintomatología comórbida en la relación entre la severidad del autismo y el nivel de intensidad del dolor, tal como se pone de evidencia en diversas publicaciones recientes (Gerrits et al., 20I4; Probst et al., 20I6; Wongpakaran et al., 2016).
En síntesis, estos resultados ponen de relieve la necesidad de identificar el dolor por medios objetivos, tanto escalares como observacionales, y tener en cuenta la psicopatología comórbida como variable moduladora de la expresión del dolor en las personas con autismo. 
Abeare, C. A. et al. (2010): "Pain, executive functioning, and affect in patients with rheumatoid arthritis". The Clinical Journal of Pain, 26 (8): 683-689.

American Psychiatric Association (2013): Diagnostic and statistical manual of mental disorders ( 5 th ed.). Washington, DC: American Psychiatric Association.

Baker, K. S. et al. (20I6): “Everyday Executive Functioning in Chronic Pain: Specific Deficits in Working Memory and Emotion Control, Predicted by Mood, Medications, and Pain Interference". The Clinical Journal of Pain, 32 (8): $673-680$.

Bandstra, N. F. et al. (2012): "Self-reported and parent-reported pain for common painful events in high-functioning children and adolescents with autism spectrum disorder”. The Clinical Journal of Pain, 28 (8): 7I 5-72I.

Baum, S. H. et al. (20I 5): "Behavioral, perceptual, and neural alterations in sensory and multisensory function in autism spectrum disorder”. Progress in Neurobiology, I34: I $40-160$.

Bento, S. et al. (2017): "Executive functions, coping, and acute pain: Emotion-focused avoidance mediates the relation between executive functioning and reports of painrelated anxiety in children". The Journal of Pain, I 8 (4): $\mathrm{S}_{3} 8$.

Bird, G. et al. (20I0): "Empathic brain responses in insula are modulated by levels of alexithymia but not autism". Brain, I33 (Pt 5):I 5 I 5-I 525 .

Bishop-Fitzpatrick, L. et al. (201 5): “The relationship between stress and social functioning in adults with autism spectrum disorder and without intellectual disability”. Autism Research, 8 (2): I64-I73.

Boucher, J. M. (2017): Autism Spectrum Disorder: Characteristics, Causes and Practical Issues. London: Sage.
Brown, W. S. y Paul, L. K. (20I5): "Brain Connectivity and the Emergence of Capacities of Personhood: Reflections from Callosal Agenesis and Autism", en Jeeves, M. (ed.): The Emergence of Personhood: A Quantum Leap? Grand Rapids, Michigan: Eerdmans Publishing Company.

Brugha, T. S. et al. (2016): "Epidemiology of autism in adults across age groups and ability levels". The British Journal of Psychiatry, 209 (6): 498-503.

Burguess, P. W. et al. (I998): “The ecological validity of tests of executive function". Journal of the International Neuropsychological Society, 4: 547-558.

Cascio, C. et al. (2008): “Tactile perception in adults with autism: a multidimensional psychophysical study". Journal of Autism and Developmental Disorders, 38: I27-I37.

Croen, L. A. et al. (2015): "The health status of adults on the autism spectrum". Autism, I9 (7): 8I4-823.

Della Fina, V. y Cera, R. (2OI 5 ): Protecting the Rights of People with Autism in the Fields of Education and Employment. Roma: SpringerVerlag.

Demetriou, E. A. et al. (2017): "Autism spectrum disorders: a meta-analysis of executive function”. Molecular Psychiatry, o०: I-7.

Duerden, E. G. et al. (2OI 5 ): “Decreased sensitivity to thermal stimuli in adolescents with autism spectrum disorder: Relation to symptomatology and cognitive ability". The Journal of Pain: Official Journal of the American Pain Society, I6 (5): 463-47I.

Eccleston, C. (I995): “The attentional control of pain: Methodological and theoretical concerns". Pain, 63 (I): 3-10.

Fan Y. T. et al. (20I3): "Empathic arousal and social understanding in individuals with autism: evidence from fMRI and ERP measurements". 
Social Cognitive and Affective Neuroscience, 9 (8): I $203-\mathrm{I} 2 \mathrm{I} 3$.

Friedman, N. P. y Miyake, A. (20I7): "Unity and diversity of executive functions: Individual differences as a window on cognitive". Cortex, 86:1 86-204.

Fusar-Poli, L. et al. (2017): "Long-term outcome of a cohort of adults with autism and intellectual disability: A pilot prospective study". Research in Developmental Disabilities, 60: 223-23 I.

García-Villamisar, D. y Rojahn, J. (2015): "Comorbid psychopathology and stress mediate the relationship between autistic traits and repetitive behaviours in adults with autism". Journal of Intellectual Disability Research, 59 (2): I I6-I 24.

Gerrits, M. M. et al. (20I4): Pain and the onset of depressive and anxiety disorders. Pain, I 55 (I): 53-59.

Gillberg, C. et al. (I985): “A 'new' chromosome marker common to the Rett syndrome and infantile autism? The frequency of fragile sites at X p22 in 8I children with infantile autism, childhood psychosis and the Rett syndrome". Brain Dev, 7 (3): 365-367.

Grinker, R. R. (20I5): "Reframing the Science and Anthropology of Autism". Culture, medicine and psychiatry, 39 (2): 345-350.

Groden J. et al. (2006): “Assessment and coping strategies”, en Baron, M. G. et al. (eds.): Stress and Coping in Autism. Nueva York: Oxford University Press.

Groden J. et al. (200I): “The development of a stress survey schedule for persons with autism and other developmental disabilities". Journal of Autism and Developmental Disorders, 3 I: 207-217.

Happé, F. y Charlton, R. A. (2012): "Aging in autism spectrum disorders: a mini-review”. Gerontology, 58 (I): 70-78.

Hayes, A. F. (2013): Introduction to mediation, moderation, and conditional process analysis: A regression-based approach. Nueva York: Guilford Press.

Hechler, T. et al. (2016): "Why harmless sensations might hurt in individuals with chronic pain:
About heightened prediction and perception of pain in the mind". Frontiers in psychology, 7: I 638 .

Howlin, P. y Magiati, I. (20I7): “Autism spectrum disorder: outcomes in adulthood". Current opinion in psychiatry, 30 (2): 69-76.

Howlin, P. y Moss, P. (20 I2): "Adults with autism spectrum disorders". The Canadian Journal of Psychiatry, 57 (5): 275-283.

Hufnagel, C. et al. (2017): “The Need for Objective Measures of Stress in Autism". Frontiers in psychology, 8, 64 .

Karsdorp, P. et al. (2013): "Response inhibition predicts painful task duration and performance in healthy individuals performing a cold pressor task in a motivational context". European Journal of Pain, I 8 (I): 92-Io0.

Kern, J. K. et al. (2006): "The pattern of sensory processing abnormalities in autism". Autism, Iо (5): 480-494.

Klintwall, L. et al. (20I I): "Sensory abnormalities in autism: a brief report". Research in developmental disabilities, 32 (2): 795-800.

Latham, J. y Davis, B. D. (I994): “The socioeconomic impact of chronic pain". Disability and rehabilitation, I6 (I): 39-44.

Loomes, R. et al. (20I7): "What Is the Male-toFemale Ratio in Autism Spectrum Disorder? A Systematic Review and Meta-Analysis”. Journal of the American Academy of Child \& Adolescent Psychiatry, 56 (6): 466-474.

Lotan, M. et al. (20I0): "Measurement properties of the Non-Communicating Adult Pain Checklist (NCAPC): a pain scale for adults with Intellectual and Developmental Disabilities, scored in a clinical setting". Research in developmental disabilities, 3 I (2): 367-375.

Mannix, M. (2016): "Medical comorbidities in autism spectrum disorder". The Brown University Child and Adolescent Behavior Letter, 32 (2): I-7.

Masiliūnas, R. et al. (2017): "Response inhibition, set shifting, and complex executive function in patients with chronic lower back pain”. Medicine, 53 (I): 26-33. 
Matson, J. L. et al. (2008): "The validity of the autism spectrum disorders-diagnosis for intellectually disabled adults (ASD-

DA)". Research in Developmental Disabilities, 29 (6): 537-546.

Matson, J. L. et al. (2007): "Norms and cut off scores for the autism spectrum disorders diagnosis for adults (ASD-DA) with intellectual disability". Research in Autism Spectrum Disorders, I (4): 330-338.

Mazefsky, C. A. y White, S. W. (20I4): "Emotion regulation: Concepts \& practice in autism spectrum disorder". Child and adolescent psychiatric clinics of North America, 23 (I): I 5-24.

Militerni, R. et al. (2000): "Pain reactivity in children with autistic disorder". The Journal of Headache and Pain, I (I): 53-56.

Minshew, N. J. y Hobson, J. A. (2008): “Sensory sensitivities and performance on sensory perceptual tasks in high-functioning individuals with autism". Journal of autism and developmental disorders, 38 (8): I485-I 498.

Moore, D. J. (20I5): “Acute pain experience in individuals with autism spectrum disorders: A review". Autism: The International Journal of Research and Practice, I9(4), 387-399.

Moriarty, O. et al. (20I I): "The effect of pain on cognitive function: a review of clinical and preclinical research". Progress in neurobiology, 93 (3): 385-404.

Nader, R. et al. (2004): "Expression of pain in children with autism". The Clinical journal of pain, 20 (2): 88-97.

Nes, L. S. et al. (2009): "Executive functions, selfregulation, and chronic pain: a review". Annals of Behavioral Medicine, 37 (2): I73-I83.

Oliver, C. et al. (20I7): "Self-injurious behaviour in people with intellectual disability and autism spectrum disorder". Current opinion in psychiatry, 30 (2): 97-IOI.

Oosterman, J. M. et al. (2010): “A unique association between cognitive inhibition and pain sensitivity in healthy participants”. European Journal of Pain, I 4 (I0): 1046-1050.
Piven, J. y Rabins, P. (20I I): “Autism spectrum disorders in older adults: toward defining a research agenda". Journal of the American Geriatrics Society, 59 (I I): 2 I 5 I-2 I 55.

Postorino, V. et al. (2016): "Mood Disorders and Autism Spectrum Disorder”, en Mazzone, L. y Vitiello, B. (eds.): Psychiatric Symptoms and Comorbidities in Autism Spectrum Disorder. Springer International Publishing.

Probst, T. et al. (2016): "Depressed mood differentially mediates the relationship between pain intensity and pain disability depending on pain duration: A moderated mediation analysis in chronic pain patients". Pain Research and Management, 2016.

Rattaz, C. et al. (2016): "How do people with autism spectrum disorders (ASD) experience pain?", en Battaglia, A. A. (ed.): An Introduction to Pain and its Relation to Nervous System Disorders. John Wiley \& Sons, Ltd.

Roth, I. (20I0): The Autism Spectrum in the 2Ist Century. London: Jessica Kingsley Publishers.

Shattuck, P. T. et al. (2OI2): "Services for adults with an autism spectrum disorder". The Canadian Journal of Psychiatry, 57 (5): 28429I.

Tantam, D. (20I4): Emotional Well-being and Mental Health: A Guide for Counsellors \& Psychotherapists. London: Sage.

Thompson, T. et al. (20I6): "Is pain perception altered in people with depression? A systematic review and meta-analysis of experimental pain research". The Journal of Pain, I7 (I 2): I257I 272.

Tordjman, S. et al. (2009): "Pain reactivity and plasma $\beta$-endorphin in children and adolescents with autistic disorder". PLoS One, 4 (8): e5289.

Uljarevic, M. et al. (2016): “Anxiety in autism spectrum disorder”, en Mazzone, L. y Vitiello, B. (eds.): Psychiatric Symptoms and Comorbidities in Autism Spectrum Disorder. Springer International Publishing.

Van der Putten, A. y Vlaskamp, C. (20I I): "Pain assessment in people with profound intellectual 
and multiple disabilities; a pilot study into the use of the Pain Behaviour Checklist in everyday practice". Research in Developmental Disabilities, 32 (5): I677-I684.

Warfield, M. E. et al. (2OI 5): "Physician perspectives on providing primary medical care to adults with autism spectrum disorders (ASD)". Journal of autism and developmental disorders, 45 (7): 2209-2217.
Wongpakaran, T. et al. (20I6): "Depression and pain: testing of serial multiple mediators". Neuropsychiatric Disease and Treatment, I 2: I849.

Yasuda, Y. et al. (2016): "Sensory cognitive abnormalities of pain in autism spectrum disorder: a case-control study". Annals of general psychiatry, I 5 (I): 8. 